\title{
EXPERIMENTAL INVESTIGATIONS OF PLASMA LENS FOCUSING AND PLASMA CHANNEL TRANSPORT OF HEAVY ION BEAMS
}

\author{
A. Tauschwitz, S.S. Yu, S. Eylon, L. Reginato, W. Leemans, J.O. Rasmussen, R.O. Bangerter, \\ Lawrence Berkeley Laboratory, Berkeley, CA 94720.
}

Final focusing of ion beams and propagation in a reactor chamber are crucial questions for heavy ion beam driven Fusion. An alternative solution to ballistic quadrupole focusing, as it is proposed in most reactor studies today, is the utilization of the magnetic field produced by a high current plasma discharge. This plasma lens focusing concept relaxes the requirements for low emittance and energy spread of the driver beam significantly and allows to separate the issues of focusing, which can be accomplished outside the reactor chamber, and of beam transport inside the reactor. For focusing a tapered wall-stabilized discharge is proposed, a concept successfully demonstrated at GSI, Germany. For beam transport a laser pre-ionized channel can be used.

\section{INTRODUCTION}

Final focusing of ion beams and their propagation in a reactor chamber are crucial for heavy ion beam driven fusion power production. The concept and technical realization of these operations have strong impacts both on the design of the accelerator and the layout of the reactor chamber. Most studies today are based on ballistic focusing by magnetic quadrupole lenses. This concept suffers from the sensitivity of the final focus to the current and shape of the beam pulse that is delivered by the driving accelerator. To reduce space charge blow-up of the driver beam in the final focus region the total beam current has to be divided into several beams, each with an individual final focusing system and a separate beam port in the reactor chamber.

An alternative solution is to strip the ions to a high charge state and to space charge and current neutralize the beam completely. An external focusing force is then applied to the beam to focus and transport it to the target. One advantage of this scheme is its physical simplicity because the beam can be treated as an ensemble of independent particles in the external focusing field. To create the focusing force the magnetic field of a high current plasma discharge can be used. This plasma lens focusing relaxes the requirements on emittance and energy spread of the driver beam significantly. A further advantage of this concept is the high insensitivity to the driver beam current and pulse shape.

The problems of focusing and of transport to the target are to a great extend independent of each other. For an experimental approach to evaluate the feasibility of plasma lens focusing these issues can be separated. Focusing of the ion beam can be accomplished using a wall-stabilized discharge. For the final transport through the reactor chamber with a radius of 2-3 meter the focused beam can be confined in a discharge channel. Since the beam is expected to be completely space charge and current neutralized in the discharge channel the whole beam intensity required to ignite a target can be transported in one channel or, if higher symmetry of the target illumination is necessary, in two channels. For a Bi Ion beam of $10 \mathrm{GeV}$ a plasma current of approximately 100 $\mathrm{kA}$ is sufficient to focus the beam and transport it to the target. Several schemes are possible to form the discharge channel inside the reactor chamber. All of them include a laser to pre-form a guiding path for the discharge channel.

A schematic layout of a reactor chamber using the proposed plasma lens final focusing is sketched in Figure 1. A single laser can be used to provide four channels from the reactor wall to the fusion pellet. Two of these channels are used to transport the driver beam and to illuminate the target from two sides. The remaining two channels are necessary to provide a return path for the discharge current. The discharge can be driven by one or several capacitor banks close to the reactor wall. To focus the driver beams down to the size of the discharge channel two wall-stabilized, tapered discharges are used. The angular acceptance of these lenses is large enough to combine several driver beams in each discharge and to keep a free optical path for the laser.

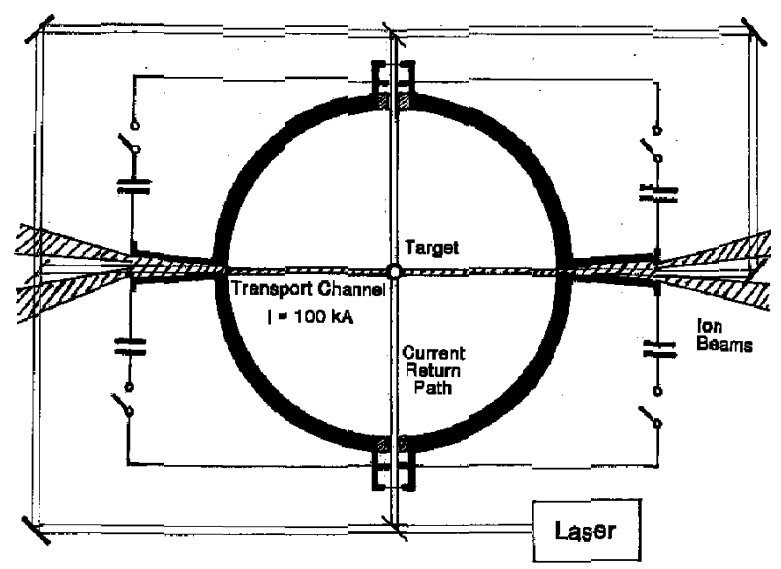

Figure 1. Schematic layout of a reactor chamber.

\section{FOCUSING WITH AN ADIABATIC PLASMA LENS}

In a plasma lens the purely azimuthal magnetic field of a gas discharge is used to focus an ion beam moving along the axis of the discharge. Since a strong, first order focusing force is applied to the beam in both transversal planes perpendicular to the beam direction this kind of focusing is much stronger than quadrupole focusing, which is, applied to both directions, an effect of only second order [1].

Plasma lenses have been developed and investigated for several years at the German Heavy Ion Laboratory GSI to 
concentrate high beam intensities onto small focal spots. The most promising results were achieved with a wall-stabilized discharge type [2]. The setup for these experiments is shown in figure 2.

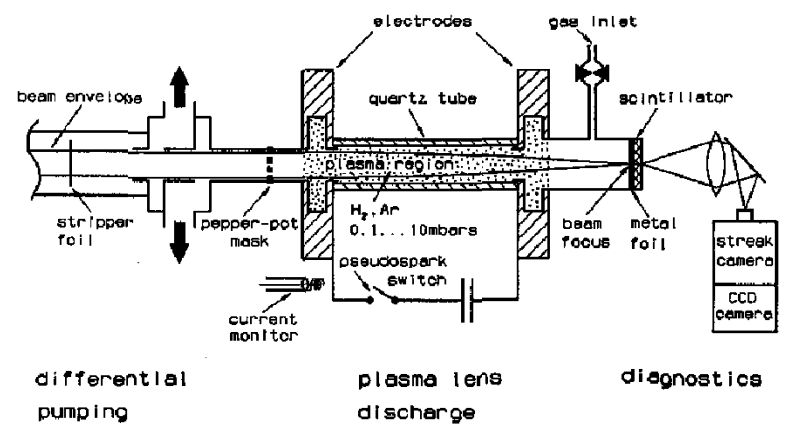

Figure 2. Setup of focusing experiments with a wallstabilized plasma lens at GSI.

A heavy ion beam is traversing a differential pumping system and is then focused in a wall-stabilized discharge. The typical beam energy for these experiments was 11.4 $\mathrm{MeV} / \mathrm{amu}$. Discharge current and length of the lens are matched to produce less than half a betatron oscillation of the ions in the lens, that means that the lens is used as a thin lens. The focus is formed a short distance downstream of the lens and is detected by a combination of a plastic scintillator and a streak camera. The wall stabilized discharge has proven better linearity of the focusing fields than a z-pinch discharge and it provides the additional opportunity to taper the discharge tube and increase the focusing strength along the lens in this way. Tests of a tapered discharge have shown that a beam with $10 \mathrm{~mm}$ initial diameter can be focused to a spot of $160 \mu \mathrm{m}$. The tapered lens has the same degree of linearity and an approximately 50\% higher focusing power than a cylindrical discharge [3].

For application of the plasma lens concept to the final focus problem in inertial confinement fusion the lens has to work as a thick lens, so that the particles perform one or more complete betatron oscillations inside the discharge. The particle trajectories in the lens are not necessarily coherent. They deviate the more from the coherent case, the broader the momentum and charge state distribution in the beam are and the more oscillations the particles perform in the lens. In the extreme case the beam fills the aperture homogeneously everywhere in the lens. Focusing can only be accomplished by increasing the focusing strength along the lens in this case. An easy way to achieve this is to taper the discharge tube diameter. To avoid beam losses the tapering has to be done adiabatically, which means that the change in betatron wavelength per betatron period has to be small.

In a focusing field a particle with coordinate $x$ satisfies the equation of motion (Hill's Equation) [4]:

$$
\frac{d^{2} x}{d z^{2}}=-k^{2} x
$$

with

$$
k^{2}(z)=\frac{Z(z)}{a^{2}(z)} \frac{2 e I}{b m c^{3}} .
$$

Here $Z$ denotes the charge state of the ion, $I$ is the total current in the lens, and $a$ is the radius of the focusing discharge. In the adiabatic approximation

$$
\frac{1}{k^{2}} \frac{d k}{d z}<<1
$$

a solution can be written in the form:

$$
x=\frac{C}{k^{1 / 2}} \exp \left(i \int k d z\right) .
$$

These equations show that the radius $a$ has to be decreased by a factor of $b^{2}$ to reduce the radius of the beam envelope $x$ by a factor of $b$. Using typical numbers for a driver beam with $m=200 \mathrm{amu}, \beta=0.3, Z=64$, and a current $I=100 \mathrm{kA}$ in a channel that is tapered down over the length of one betatron wavelength at the entrance of the lens of $\lambda_{\beta}=$ $152 \mathrm{~cm}$ from $20 \mathrm{~mm}$ to $5 \mathrm{~mm}$ radius yields a reduction of the beam radius from $R=10 \mathrm{~mm}$ to $5 \mathrm{~mm}$. The admissible emittance for the beam is determined by the conservation of phase space density over the simple relation

$$
\frac{\varepsilon}{R}=k a,
$$

which allows a normalized emittance of $125 \mathrm{~mm} \mathrm{mrad}$ at the end of the lens for the example.

An experiment is designed to investigate this adiabatic focusing experimentally using the $2 \mathrm{MeV}$ beam from the LBL-ESQ injector. The schematic layout for this experiment is sketched in figure 3. The beam traverses a two stage differential pumping system capable to separate the discharge gas of approximately 1 Torr from the accelerator vacuum of $10^{-6}$ Torr. The discharge tube radius is tapered from $10 \mathrm{~mm}$ to $2.5 \mathrm{~mm}$ to reduce the beam radius from $5 \mathrm{~mm}$ to $2.5 \mathrm{~mm}$. The intensity distribution in the focused beam will be scanned by a pinhole close to the end of the discharge and measured with a Faraday cup.

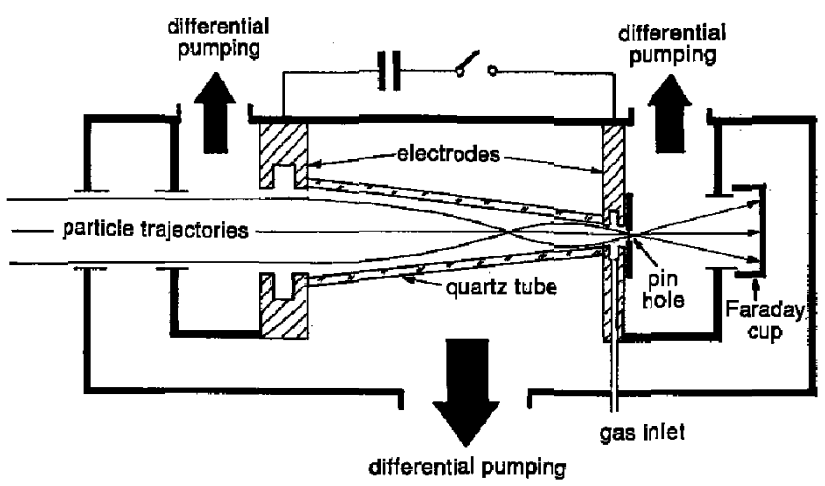

Figure 3. Setup for focusing experiments with an adiabatic plasma lens at the LBL ESQ-injector.

The beam is expected to be in an average charge state between 2 and 3 [5]. With a discharge length of $30 \mathrm{~cm}$ and a 
discharge current of $20 \mathrm{kA}$ the particles perform one and a half betatron oscillations in the lens so that an initially parallel beam of low emittance forms one focal spot inside the lens and another one at the end of the lens. The calculated emittance that this lens can handle without loosing particles is $225 \mathrm{~mm}$ mrad. This number is consistent with Monte-Carlo simulations of the focusing. These calculations include charge transfer processes instead of simply using an averaged charge state but do not include scattering of the ions in the discharge plasma. For the above mentioned case these calculations yield a negligible amount of particle losses below an emittance of $225 \mathrm{~mm}$ mrad and an almost linear increase of particle losses to about $35 \%$ for an emittance of $500 \mathrm{~mm}$ mrad.

At the low energy of $2 \mathrm{MeV}$ scattering in the discharge plasma is an important factor. To determine the emittance increase by scattering the scattering in a $2 \mathrm{~cm}$ gas stripper cell has been measured for helium, nitrogen, and argon gas of up to 1 Torr pressure. From these measurements scattering angles up to $100 \mathrm{mrad}$ can be expected for $0.9 \mathrm{MeV}$ beam energy at the end of a $30 \mathrm{~cm}$ long tube with 1 Torr helium. This amounts to approximately $250 \mathrm{~mm} \mathrm{mrad}$ emittance of the beam at the end of the focusing discharge, which is only slightly above the tolerable emittance. For higher beam energies or lower gas pressures no beam losses due to scattering are expected.

\section{BEAM TRANSPORT IN A DISCHARGE CHANNEL}

The driver beams can be combined and focused to a diameter of $10 \mathrm{~mm}$ outside the reactor. To keep the beam at this size during the 2 or $3 \mathrm{~m}$ radius of the reactor chamber the same magnetic field is required as at the end of the focusing lens. For the above mentioned example this can be achieved by a discharge channel of $10 \mathrm{~mm}$ diameter with $100 \mathrm{kA}$ current. To guide such a channel from the beam port in the reactor wall to the target a laser can be used to pre-ionize the background gas in the reactor chamber.

Several coupling mechanisms of the laser energy to the gas have been studied experimentally. One of the most efficient ways is to add a small percentage of an organic molecule with a high photo ionization crossection to the gas and to photo-ionize these organic molecules in a two photon process with an excimer laser. Breakdown of a high current discharge over up to $1.2 \mathrm{~m}$ along a laser path has been reported at $30 \%$ of the self breakdown voltage of the system [6]. For a reactor chamber a sufficient pre-ionization can be achieved with an excimer laser of 1 to $10 \mathrm{~J}$ pulse energy.

A crucial point in this transport scheme is the stability of the discharge channel. Unless very high voltages are used to create the channel, the high inductance of the long current path through the reactor chamber will limit the current rise time to several microseconds. The highest hydrodynamic stability of the plasma channel is expected for a discharge in a heavy gas as argon at a pressure of 10 Torr or more. An experiment is set up to address the problems of dynamics and stability for a discharge channel of up to $90 \mathrm{~cm}$ length with a discharge current in the range of 50 to $100 \mathrm{kA}$ and a current rise time of four microseconds. Further objectives of the experiment are the interaction of the plasma channel with a target and possible interactions between the channel and it's return current path.

\section{CONCLUSION}

Final focusing based on plasma lens focusing and discharge channel transport seems feasible if the stability of the channel is sufficient and if the problem of channel-target interaction can be solved. The advantages of this focusing scheme for the driving accelerator are relaxed requirements for emittance and momentum spread. The advantage for the reactor layout is the much smaller number and area of beam ports in the reactor wall, which makes the problem of explosion debris flying up the beam lines much easier to solve.

\section{ACKNOWLEDGMENTS}

One of the authors (A.T.) wishes to thank the German Humboldt foundation for granting a Feodor-Lynen fellowship to support his visit at the Lawrence Berkeley Laboratory.

\section{REFERENCES}

[1] E. Boggasch, B. Heimrich, D.H.H. Hoffmann, "Focusing behaviour of plasma lenses compared to conventional quadrupole systems", Nucl. Instr. Meth. A 336 (1993) 438-441.

[2] E. Boggasch, A. Tauschwitz, H. Wahl, K.-G. Dietrich, D.H.H. Hoffmann, W. Laux, M. Stetter, R. Tkotz, "Plasma lens fine focusing of heavy-ion beams", Appl. Phys. Lett. 60 (1992) 2475-2477.

[3] A. Tauschwitz, M. de Magistris, E. Boggasch, M. Dornik, D.H.H. Hoffmann, J. Jacoby, W. Seelig, P. Spiller, H. Wetzler, "Performance of a Shape Optimized Conical Plasma Lens", GSI Report 94-10 (1994) 10

[4] P. Chen, K. Oide, A.M. Sessler, S.S. Yu, "Plasma-Based Adiabatic Focuser", Phys. Rev. Lett. 64 (1990) 1231-1234

[5] H.D. Betz, "Heavy Ion Charge States", in Applied Atomic Collision Physics, Pure and Applied Physics, 43-4 (1983) 1-42

[6] C.A. Frost, J.R. Woodworth, J.N. Olsen, T.A. Green, "Plasma channel formation with ultraviolet lasers", Appl. Phys. Lett. 41 (1982) 813-815 